\title{
The peak statistics on simsun permutations
}

\author{
Shi-Mei Ma* \\ School of Mathematics and Statistics \\ Northeastern University at Qinhuangdao \\ Hebei 066000, P.R. China \\ shimeimapapers@163.com
}

\author{
Yeong-Nan Yeh $^{\dagger}$ \\ Institute of Mathematics \\ Academia Sinica \\ Taipei, Taiwan \\ mayeh@math.sinica.edu.tw
}

Submitted: Feb 1, 2016; Accepted: Apr 2, 2016; Published: Apr 15, 2016

Mathematics Subject Classifications: 05A05, 05A15

\begin{abstract}
In this paper, we study the relationship among left peaks, interior peaks and up-down runs of simsun permutations. Properties of the generating polynomials, including the recurrence relation, generating function and real-rootedness are studied. Moreover, we introduce and study simsun permutations of the second kind.
\end{abstract}

Keywords: Simsun permutations; Left peaks; Interior peaks; Alternating runs; Excedances

\section{Introduction}

Let $\mathfrak{S}_{n}$ denote the symmetric group of all permutations of $[n]$, where $[n]=\{1,2, \ldots, n\}$. Let $\pi=\pi(1) \pi(2) \cdots \pi(n) \in \mathfrak{S}_{n}$. A descent of $\pi$ is an index $i \in[n-1]$ such that $\pi(i)>\pi(i+1)$. We say that $\pi$ has no double descents if there is no index $i \in[n-2]$ such that $\pi(i)>\pi(i+1)>\pi(i+2)$. The permutation $\pi$ is called simsun if for all $k$, the subword of $\pi$ restricted to $[k]$ (in the order they appear in $\pi$ ) contains no double descents. For example, 35142 is a simsun permutation, but 35241 is not. Simsun permutations are useful in describing the action of the symmetric group on the maximal chains of the partition lattice (see [24, 25]). They are a variant of André permutations introduced by Foata and Schützenberger [12]. There has been much recent work related to simsun permutations (see $[3,5,8,11,13,15]$ for instance).

Let $a_{i}(n)$ be the number of distinct $S_{n}$-orbits such that the stabiliser of a maximal chain in the orbit is conjugate to the Young subgroup $S_{2}^{i} \times S_{1}^{n-2 i}$. Following Sundaram [24,

\footnotetext{
*Supported by NSFC (11401083) and the Fundamental Research Funds for the Central Universities (N152304006).

${ }^{\dagger}$ Supported by NSC 101-2115-M-001-013-MY3.
} 
Theorem 3.2], the numbers $a_{i}(n)$ satisfy the recurrence relation

$$
a_{i}(n+1)=i a_{i}(n)+(n-2 i+2) a_{i-1}(n),
$$

with initial conditions $a_{0}(1)=1=a_{1}(2), a_{0}(n)=0$ for $n>1$ and $a_{i}(n)=0$ if $2 i>n$. Let $|C|$ denote the cardinality of a set $C$. The descent number of $\pi \in \mathfrak{S}_{n}$ is defined by $\operatorname{des}(\pi)=|\{i \in[n-1]: \pi(i)>\pi(i+1)\}|$. Let $\mathcal{R S}_{n}$ be the set of simsun permutations of length $n$. Simion and Sundaram [24, p. 267] discovered that $a_{i}(n)$ is the number of permutations in $\mathcal{R} \mathcal{S}_{n-2}$ with $i-1$ descents and $\left|\mathcal{R} \mathcal{S}_{n}\right|=E_{n+1}$, where $E_{n}$ is the $n$th Euler number, which also is the number alternating permutations in $\mathfrak{S}_{n}$. Let

$$
S(n, k)=\left|\left\{\pi \in \mathcal{R} \mathcal{S}_{n}: \operatorname{des}(\pi)=k\right\}\right| .
$$

In other words, let $S(n, k)=a_{k+1}(n+2)$. We define $S_{n}(x)=\sum_{k=0}^{\lfloor n / 2\rfloor} S(n, k) x^{k}$. It follows from (1) that the numbers $S(n, k)$ satisfy the recurrence relation

$$
S(n, k)=(k+1) S(n-1, k)+(n-2 k+1) S(n-1, k-1),
$$

with the initial conditions $S(0,0)=1$ and $S(0, k)=0$ for $k \geqslant 1$, which is equivalent to

$$
S_{n+1}(x)=(1+n x) S_{n}(x)+x(1-2 x) S_{n}^{\prime}(x),
$$

with $S_{0}(x)=1$. Let $R S(x, z)=\sum_{n \geqslant 0} S_{n}(x) \frac{z^{n}}{n !}$. Chow and Shiu [5, Theorem 2.1] obtained that

$$
R S(x, z)=\left(\frac{\sqrt{2 x-1} \sec \left(\frac{z}{2} \sqrt{2 x-1}\right)}{\sqrt{2 x-1}-\tan \left(\frac{z}{2} \sqrt{2 x-1}\right)}\right)^{2} .
$$

For convenience, here we list the first few terms of $S_{n}(x)$ :

$S_{1}(x)=1, S_{2}(x)=1+x, S_{3}(x)=1+4 x, S_{4}(x)=1+11 x+4 x^{2}, S_{5}(x)=1+26 x+34 x^{2}$.

The number of peaks of permutations is certainly among the most important combinatorial statistics. See, e.g., $[2,16,18]$ and the references therein. A left peak in $\pi$ is an index $i \in[n-1]$ such that $\pi(i-1)<\pi(i)>\pi(i+1)$, where we take $\pi(0)=0$. Let lpk $(\pi)$ denote the number of left peaks in $\pi$. For example, lpk $(21435)=2$. Sundaram discovered that $a_{i}(n)$ is also the number of André permutations in $\mathfrak{S}_{n-1}$ with $i$ left peaks (see $[26$, p. 175]). In fact, since any descent of a simsun permutation is a left peak, we have

$$
S_{n}(x)=\sum_{\pi \in \mathcal{R} \mathcal{S}_{n}} x^{\mathrm{lpk}(\pi)}
$$

Let $\widehat{W}(n, k)=\left|\left\{\pi \in \mathfrak{S}_{n}: \operatorname{lpk}(\pi)=k\right\}\right|$. Let $\widehat{W}_{n}(x)=\sum_{k \geqslant 0} \widehat{W}(n, k) x^{k}$. The polynomials $\widehat{W}_{n}(x)$ satisfy the recurrence relation

$$
\widehat{W}_{n+1}(x)=(1+n x) \widehat{W}_{n}(x)+2 x(1-x) \widehat{W}_{n}^{\prime}(x),
$$


with initial conditions $\widehat{W}_{0}(x)=\widehat{W}_{1}(x)=1$ (see [16] for instance). Following [21, A008971] the exponential generating function of $\widehat{W}_{n}(x)$ is given as follows:

$$
\widehat{W}(x, z)=\sum_{n \geqslant 0} \widehat{W}_{n}(x) \frac{z^{n}}{n !}=\frac{\sqrt{1-x}}{\sqrt{1-x} \cosh (z \sqrt{1-x})-\sinh (z \sqrt{1-x})} .
$$

By comparing (4) with (5), we observe that $R S(x, z)=\widehat{W}^{2}(2 x, z / 2)$, which leads to the following formula:

$$
S_{n}(x)=\frac{1}{2^{n}} \sum_{k=0}^{n}\left(\begin{array}{l}
n \\
k
\end{array}\right) \widehat{W}_{k}(2 x) \widehat{W}_{n-k}(2 x) .
$$

Denote by $B_{n}$ the hyperoctahedral group of rank $n$. The elements $\pi$ of $B_{n}$ are the signed permutations of the set $\pm[n]$ such that $\pi(-i)=-\pi(i)$ for all $i$, where $\pm[n]=$ $\{ \pm 1, \pm 2, \ldots, \pm n\}$. A snake of type $B_{n}$ is a signed permutation $\pi(1) \pi(2) \cdots \pi(n)$ of $B_{n}$ such that $0<\pi(1)>\pi(2)<\cdots \pi(n)$. The $n$th Springer number $s_{n}$ is the number of snakes of type $B_{n}$. Springer [22] derived the following generating function:

$$
s(z)=\sum_{n \geqslant 0} s_{n} \frac{z^{n}}{n !}=\frac{1}{\cos z-\sin z} .
$$

Recall that $S_{n}(1)=E_{n+1}$. It is clear that $\widehat{W}(2, z)=s(z)$. Therefore, as a special case of (6), we get

$$
E_{n+1}=\frac{1}{2^{n}} \sum_{k=0}^{n}\left(\begin{array}{l}
n \\
k
\end{array}\right) s_{k} s_{n-k} .
$$

We refer the reader to [4] for various structures related to Springer numbers. Motivated by (6), it is natural to further study peak statistics on simsun permutations.

This paper is organized as follows. In Section 2, we give a constructive proof of a connection between $S(n, k)$ and the number of permutations in $\mathfrak{S}_{n+1}$ with $k$ interior peaks. In Section 3, we count simsun permutations by their interior peaks. In Section 4 , we count simsun permutations by their up-down runs. In Section 5, we introduce simsun permutations of the second kind.

\section{Relationship to interior peaks of permutations in $\mathfrak{S}_{n}$}

We first recall some basic definitions of peak statistics. An interior peak in $\pi$ is an index $i \in\{2,3, \ldots, n-1\}$ such that $\pi(i-1)<\pi(i)>\pi(i+1)$. Let pk $(\pi)$ denote the number of interior peaks in $\pi$. For example, $\operatorname{pk}(21435)=1$. Let $W(n, k)$ be the number of permutations in $\mathfrak{S}_{n}$ with $k$ interior peaks and let $W_{n}(x)=\sum_{k \geqslant 0} W(n, k) x^{k}$. We say that $\pi$ changes direction at position $i$ if either $\pi(i-1)<\pi(i)>\pi(i+1)$, or $\pi(i-1)>\pi(i)<\pi(i+1)$, where $i \in\{2,3, \ldots, n-1\}$. We say that $\pi$ has $k$ alternating runs if there are $k-1$ indices $i$ where $\pi$ changes direction (see [1]). Let $R(n, k)$ denote the number of permutations in $\mathfrak{S}_{n}$ with $k$ alternating runs and let $R_{n}(x)=\sum_{k=1}^{n-1} R(n, k) x^{k}$. 
Let $d(n, k)$ denote the number of increasing $0-1-2$ trees on $[n]$ with $k$ leaves (see $[21$, A094503]). Let $D_{n}(x)=\sum_{k \geqslant 1} d(n, k) x^{k}$.

It follows from [5, Proposition 4] that $D_{n+1}(x)=x S_{n}(x)$ for $n \geqslant 0$. Using [18, Corollary 2, Theorem 11], we get

$$
R_{n}(x)=\frac{x(1+x)^{n-2}}{2^{n-2}} W_{n}\left(\frac{2 x}{1+x}\right)=2 x(1+x)^{n-2} S_{n-1}\left(\frac{x}{1+x}\right) \quad \text { for } n \geqslant 2 .
$$

Combining (7) and [17, Eq. (13)], we get the following result.

Proposition 1. For $n \geqslant 1$ and $0 \leqslant k \leqslant\left\lfloor\frac{n}{2}\right\rfloor$, we have

$$
W(n+1, k)=2^{n-k} S(n, k) .
$$

Let $\left\{\begin{array}{c}n \\ i\end{array}\right\}$ be the Stirling number of the second kind and let

$$
p(n, n-2 k+1)=(-1)^{k} \sum_{i \geqslant 1} i !\left\{\begin{array}{l}
n \\
i
\end{array}\right\}(-2)^{n-i}\left[\left(\begin{array}{c}
i \\
n-2 k
\end{array}\right)-\left(\begin{array}{c}
i \\
n-2 k+1
\end{array}\right)\right] .
$$

Then

$$
S_{n}(x)=\frac{1}{2^{n+1} x} \sum_{k=0}^{\lfloor n / 2\rfloor+1} p(n+1, n-2 k+2)(2 x-1)^{k} \quad \text { for } n \geqslant 1 .
$$

In the rest of this section, we give a constructive proof of (8). Let

$$
D(\pi)=\{i \in[n-2]: \pi(i)>\pi(i+1)\}
$$

be the descent set of $\pi \in \mathcal{R} \mathcal{S}_{n}$. It should be noted that if we get a permutation $\pi^{\prime} \in \mathcal{R} \mathcal{S}_{n+1}$ from a permutation $\pi \in \mathcal{R} \mathcal{S}_{n}$ by inserting the entry $n+1$ into $\pi$, then the entry $n+1$ cannot be inserted before $\pi(i)$, where $i \in D(\pi)$. In the following discussion, we always assume that all permutations in $\mathcal{R S}_{n}$ are prepended by 0 . That is, we identify a permutation $\pi(1) \pi(2) \cdots \pi(n) \in \mathcal{R} \mathcal{S}_{n}$ with the permutation $\pi(0) \pi(1) \pi(2) \cdots \pi(n)$, where $\pi(0)=0$. Define $\mathcal{R S}_{n, k}=\left\{\pi \in \mathcal{R} \mathcal{S}_{n} \mid \operatorname{des}(\pi)=k\right\}$. Set $\overleftarrow{D}(\pi)=\{i-1: i \in D(\pi)\}$. We can now introduce a definition of the labeled simsun permutations.

Definition 2. Let $\pi \in \mathcal{R S}_{n, k}$. Suppose $i_{1}<i_{2}<\cdots<i_{k}$ are elements of $D(\pi)$. Then we put the superscript label $x_{r}$ right after $\pi\left(i_{r}\right)$, where $1 \leqslant r \leqslant k$. If $j_{1}<j_{2}<\cdots<j_{n-2 k}$ are elements of the set $\{0,1,2, \ldots, n-1\} \backslash(D(\pi) \cup \overleftarrow{D}(\pi))$, then we put the superscript label $y_{s}$ right after $\pi\left(j_{s}\right)$, where $1 \leqslant s \leqslant n-2 k$.

Let $\mathfrak{S}_{n, k}=\left\{\pi \in \mathfrak{S}_{n} \mid \operatorname{pk}(\pi)=k\right\}$. We introduce a definition of labeled permutations.

Definition 3. Let $\pi \in \mathfrak{S}_{n, k}$. Suppose $i_{1}<i_{2}<\cdots<i_{k}$ are indices of the interior peaks of $\pi$. Then we put the superscript labels $p_{r}$ immediately before and right after $\pi\left(i_{r}\right)$, where $1 \leqslant r \leqslant k$. If $j_{1}<j_{2}<\cdots<j_{n-2 k-1}$ are elements of the set $\{1,2,3, \ldots, n-1\} \backslash$ $\left(\left\{i_{1}, i_{2}, \ldots, i_{k}\right\} \cup\left\{i_{1}-1, i_{2}-1, \ldots, i_{k}-1\right\}\right)$, then we put the superscript label $q_{s}$ right after $\pi\left(j_{s}\right)$, where $1 \leqslant s \leqslant n-2 k-1$. 
In the following, we shall add labels to permutations in $\mathcal{R} \mathcal{S}_{n, k}$ and $\mathfrak{S}_{n, k}$. As an example, for $\pi=34125$, if we say that $\pi \in \mathcal{R S}_{5,1}$, then the labeling of $\pi$ is given by ${ }^{y_{1}} 34^{x_{1}} 1^{y_{2}} 2^{y_{3}} 5$; if we say that $\pi \in \mathfrak{S}_{5,1}$, then the labeling of $\pi$ is given by $3^{p_{1}} 4^{p_{1}} 1^{q_{1}} 2^{q_{2}} 5$.

Now we construct a correspondence, denoted by $\Phi$, between $\mathcal{R} \mathcal{S}_{n, k}$ and $\mathfrak{S}_{n+1, k}$. When $n=1$, the correspondence between $\mathcal{R} \mathcal{S}_{1,0}$ and $\mathfrak{S}_{2,0}$ is given by

$$
y_{1} 1 \stackrel{\Phi}{\longleftrightarrow}\left\{1^{q_{1}} 2,2^{q_{1}} 1\right\} .
$$

When $n=2$, the correspondence between $\mathcal{R S}_{2, k}$ and $\mathfrak{S}_{3, k}$ is given by

$$
\begin{aligned}
& y_{1} 1^{y_{2}} 2 \stackrel{\Phi}{\longleftrightarrow}\left\{1^{q_{1}} 2^{q_{2}} 3,3^{q_{1}} 1^{q_{2}} 2,2^{q_{1}} 1^{q_{2}} 3,3^{q_{1}} 2^{q_{2}} 1\right\} ; \\
& 2^{x_{1}} 1 \stackrel{\Phi}{\longleftrightarrow}\left\{1^{p_{1}} 3^{p_{1}} 2,2^{p_{1}} 3^{p_{1}} 1\right\} .
\end{aligned}
$$

Let $n=m$. Suppose $\Phi$ is a correspondence between $\mathcal{R} \mathcal{S}_{m, k}$ and $\mathfrak{S}_{m+1, k}$ for all $k$. More precisely, given an element $\pi \in \mathcal{R S}_{m, k}$. Suppose we have the correspondence

$$
\pi \stackrel{\Phi}{\longleftrightarrow}\left\{\sigma_{1}, \sigma_{2}, \ldots, \sigma_{2^{m-k}}\right\},
$$

where $\sigma_{i} \in \mathfrak{S}_{m+1, k}$ for $1 \leqslant i \leqslant 2^{m-k}$. Consider the case $n=m+1$. Suppose $\widehat{\pi} \in \mathcal{R} \mathcal{S}_{m+1}$ is obtained from $\pi$ by inserting the entry $m+1$ into $\pi$. We distinguish three cases:

(i) If $\widehat{\pi}(m+1)=m+1$, then we insert the entry $m+2$ at the front or at the end of each $\sigma_{i}$. In this case, the obtained elements in $\Phi(\widehat{\pi})$ all have $k$ interior peaks. Therefore, we get $2 \cdot 2^{m-k}=2^{m+1-k}$ elements in $\mathfrak{S}_{m+2, k}$.

(ii) If the entry $m+1$ is inserted to the position of $\pi$ with label $x_{r}$, then we insert the entry $m+2$ to one of the positions of each $\sigma_{i}$ with label $p_{r}$. In this case, des $(\widehat{\pi})=k$ and we get $2 \cdot 2^{m-k}=2^{m+1-k}$ elements in $\mathfrak{S}_{m+2, k}$.

(iii) If the entry $m+1$ is inserted to the position of $\pi$ with label $y_{s}$, then we insert the entry $m+2$ to the position of each $\sigma_{i}$ with label $q_{s}$. In this case, $\operatorname{des}(\widehat{\pi})=k+1$ and we get $2^{m-k}=2^{(m+1)-(k+1)}$ elements in $\mathfrak{S}_{m+2, k+1}$.

It is straightforward to show that each labeled permutation in $\Phi(\widehat{\pi})$ will be obtained exactly once in this way. Conversely, given an element $\tau$ of $\mathfrak{S}_{m+2, k}$. Removing the largest entry $m+2$ of $\tau$, we can find the position of the largest entry of the corresponding simsun permutation in $\mathcal{R S}_{m+1}$. As illustrated in example 4, we can get an unique element of $\mathcal{R S}_{m+1}$ by repeatedly removing the largest entry. By induction, we see that $\Phi$ is the desired correspondence between $\mathcal{R S}_{m, k}$ and $\mathfrak{S}_{m+1, k}$, which also gives a constructive proof of (8).

Example 4. Given $\pi=312 \in \mathcal{R S}_{3,1}$. The correspondence between $\pi$ and $\Phi(\pi)$ is built up as follows:

$$
\begin{aligned}
& y_{1} \stackrel{\Phi}{\longleftrightarrow}\left\{1^{q_{1}} 2,2^{q_{1}} 1\right\} ; \\
& y_{1} 1^{y_{2}} 2 \stackrel{\Phi}{\longleftrightarrow}\left\{1^{q_{1}} 2^{q_{2}} 3,3^{q_{1}} 1^{q_{2}} 2,2^{q_{1}} 1^{q_{2}} 3,3^{q_{1}} 2^{q_{2}} 1\right\} ; \\
& 3^{x_{1}} 1^{y_{1}} 2 \stackrel{\Phi}{\longleftrightarrow}\left\{1^{p_{1}} 4^{p_{1}} 2^{q_{1}} 3,3^{p_{1}} 4^{p_{1}} 1^{q_{1}} 2,2^{p_{1}} 4^{p_{1}} 1^{q_{1}} 3,3^{p_{1}} 4^{p_{1}} 2^{q_{1}} 1\right\} .
\end{aligned}
$$




\section{Interior peaks of simsun permutations}

Let $\mathcal{R S}_{n}^{+}=\left\{\pi \in \mathcal{R S}_{n}: \pi(1)>\pi(2)\right\}$ and $\mathcal{R S}_{n}^{-}=\left\{\pi \in \mathcal{R} \mathcal{S}_{n}: \pi(1)<\pi(2)\right\}$. For $\pi \in \mathcal{R S}_{n}^{+}$, we have lpk $(\pi)=\operatorname{pk}(\pi)+1$. While for $\pi \in \mathcal{R S}_{n}^{-}$, we have $\operatorname{lpk}(\pi)=\operatorname{pk}(\pi)$.

We define

$$
\begin{aligned}
P_{n}(x) & =\sum_{\pi \in \mathcal{R} \mathcal{S}_{n}} x^{\mathrm{pk}(\pi)}=\sum_{k \geqslant 0} P(n, k) x^{k}, \\
P_{n}^{+}(x) & =\sum_{\pi \in \mathcal{R} \mathcal{S}_{n}^{+}} x^{\mathrm{pk}(\pi)}=\sum_{k \geqslant 0} P^{+}(n, k) x^{k}, \\
P_{n}^{-}(x) & =\sum_{\pi \in \mathcal{R} \mathcal{S}_{n}^{-}} x^{\mathrm{pk}(\pi)}=\sum_{k \geqslant 0} P^{-}(n, k) x^{k} .
\end{aligned}
$$

The following lemma is a fundamental result.

Lemma 5. For $n \geqslant 2$, we have

$$
\begin{gathered}
P^{+}(n+1, k)=(k+1) P^{+}(n, k)+(n-2 k) P^{+}(n, k-1)+P^{-}(n, k), \\
P^{-}(n+1, k)=(k+1) P^{-}(n, k)+(n-2 k+1) P^{-}(n, k-1)+P^{+}(n, k-1) .
\end{gathered}
$$

Equivalently, the polynomials $P_{n}^{+}(x)$ and $P_{n}^{-}(x)$ satisfy the following recurrence relations

$$
\begin{aligned}
& P_{n+1}^{+}(x)=((n-2) x+1) P_{n}^{+}(x)+x(1-2 x) \frac{d}{d x} P_{n}^{+}(x)+P_{n}^{-}(x), \\
& P_{n+1}^{-}(x)=((n-1) x+1) P_{n}^{-}(x)+x(1-2 x) \frac{d}{d x} P_{n}^{-}(x)+x P_{n}^{+}(x) .
\end{aligned}
$$

Proof. We now prove (9). In order to get a permutation $\pi^{\prime} \in \mathcal{R} \mathcal{S}_{n+1}^{+}$with $k$ interior peaks from a permutation $\pi \in \mathcal{R} \mathcal{S}_{n}$, there are three ways to insert the entry $n+1$ into $\pi$ :

(a) If $\pi \in \mathcal{R S}_{n}^{+}$and pk $(\pi)=k$, then we can insert the entry $n+1$ right after an interior peak of $\pi$ or put the entry $n+1$ at the end of $\pi$. As we have $P^{+}(n, k)$ choices for $\pi$, this accounts for $(k+1) P^{+}(n, k)$ possibilities.

(b) If $\pi \in \mathcal{R S}_{n}^{+}$and pk $(\pi)=k-1$, then there are $n-2 k$ positions could be inserted the entry $n+1$, since we cannot insert the entry $n+1$ immediately before or right after each left peak of $\pi$. As we have $P^{+}(n, k-1)$ choices for $\pi$, this accounts for $(n-2 k) P^{+}(n, k-1)$ possibilities.

(c) If $\pi \in \mathcal{R S}_{n}^{-}$and $\mathrm{pk}(\pi)=k$, then we have to put the entry $n+1$ at the front of $\pi$.

This completes the proof of (9). In the same way, one can prove (10).

The first few terms of the $P_{n}(x), P_{n}^{+}(x)$ and $P_{n}^{-}(x)$ are respectively given as follows:

$$
\begin{gathered}
P_{1}(x)=1, P_{2}(x)=2, P_{3}(x)=3+2 x, P_{4}(x)=4+12 x, P_{5}(x)=5+44 x+12 x^{2} ; \\
P_{1}^{+}(x)=1, P_{2}^{+}(x)=1, P_{3}^{+}(x)=2, P_{4}^{+}(x)=3+4 x, P_{5}^{+}(x)=4+22 x ; \\
P_{1}^{-}(x)=1, P_{2}^{-}(x)=1, P_{3}^{-}(x)=1+2 x, P_{4}^{-}(x)=1+8 x, P_{5}^{-}(x)=1+22 x+12 x^{2} .
\end{gathered}
$$


By Lemma 5, it is easy to deduce that

$$
\operatorname{deg} P_{n}^{+}(x)=\lfloor(n-2) / 2\rfloor, \operatorname{deg} P_{n}(x)=\operatorname{deg} P_{n}^{-}(x)=\lfloor(n-1) / 2\rfloor .
$$

Lemma 6. For $n \geqslant 1$, we have

$$
P^{+}(n+1, k)=(n-2 k) S(n, k), P^{-}(n+1, k)=(1+k) S(n, k) .
$$

Equivalently,

$$
P_{n+1}^{+}(x)=n S_{n}(x)-2 x S_{n}^{\prime}(x), P_{n+1}^{-}(x)=S_{n}(x)+x S_{n}^{\prime}(x) .
$$

Proof. We prove (11) by induction. If $n=1$, the result is obvious, so we proceed to the inductive step. Suppose the result holds for $n=m$. For $n=m+1$, combining (2) and (9), we have

$$
\begin{aligned}
P^{+}(m+2, k) & =(k+1) P^{+}(m+1, k)+(m+1-2 k) P^{+}(m+1, k-1)+P^{-}(m+1, k) \\
& =(k+1)(m-2 k) S(m, k)+(m+1-2 k)(m+2-2 k) S(m, k-1)+ \\
& (k+1) S(m, k) \\
& =(k+1)(m-2 k) S(m, k)+(m+1-2 k)(m+2-2 k) S(m, k-1)+ \\
& S(m+1, k)-(m+2-2 k) S(m, k-1) \\
& =(m-2 k)[(k+1) S(m, k)+(m+2-2 k) S(m, k-1)]+S(m+1, k) \\
& =(m-2 k) S(m+1, k)+S(m+1, k) \\
& =(m+1-2 k) S(m+1, k) .
\end{aligned}
$$

Along the same lines, one can prove $P^{-}(n+1, k)=(1+k) S(n, k)$.

It is clear that $P(n, k)=P^{+}(n, k)+P^{-}(n, k)$ for $n \geqslant 2$. We can now conclude the following result from the discussion above.

Theorem 7. For $n \geqslant 1$, we have

$$
P(n+1, k)=(n+1-k) S(n, k),
$$

or equivalently,

$$
P_{n+1}(x)=(n+1) S_{n}(x)-x S_{n}^{\prime}(x) .
$$

Furthermore, we have

$$
P(n+1, k)=\frac{(k+1)(n-k+1)}{n-k} P(n, k)+(n-2 k+1) P(n, k-1)
$$

for $0 \leqslant k \leqslant\lfloor n / 2\rfloor$. In particular, $P(n, 0)=n$ and $P(n, 1)=(n-1)\left(2^{n-1}-n\right)$ for $n \geqslant 1$. 
It should be noted that (15) follows immediately from (2) and (13).

We now recall some notations from [14] concerning the zeros of polynomials. Let RZ denote the set of real polynomials with only real zeros. Furthermore, denote by RZ $(I)$ the set of such polynomials all of whose zeros are in the interval $I$. Suppose that $p, q \in \mathrm{RZ}$, the zeros of $p$ are $\xi_{1} \leqslant \cdots \leqslant \xi_{n}$, and the zeros of $q$ are $\theta_{1} \leqslant \cdots \leqslant \theta_{m}$. We say that $p$ interlaces $q$ if $\operatorname{deg} q=1+\operatorname{deg} p$ and the zeros of $p$ and $q$ satisfy

$$
\theta_{1} \leqslant \xi_{1} \leqslant \theta_{2} \leqslant \cdots \leqslant \xi_{n} \leqslant \theta_{n+1}
$$

We also say that $p$ alternates left of $q$ if $\operatorname{deg} p=\operatorname{deg} q$ and the zeros of $p$ and $q$ satisfy

$$
\xi_{1} \leqslant \theta_{1} \leqslant \xi_{2} \leqslant \cdots \leqslant \xi_{n} \leqslant \theta_{n}
$$

We use the notation $p \nmid q$ for " $p$ interlaces $q$," $p \ll q$ for " $p$ alternates left of $q$," and $p \prec q$ for either $p \nmid q$ or $p \ll q$. For notational convenience, let $a \prec b x+c$ for any real constants $a, b, c$.

We now recall a result on the real-rootedness of $S_{n}(x)$.

Lemma 8 ([5, Theorem 2.1]). For $n \geqslant 2$, we have $S_{n}(x) \in \mathrm{RZ}(-\infty, 0)$ and $S_{n}(x) \prec$ $S_{n+1}(x)$.

Let sgn denote the sign function defined on $\mathbb{R}$.

Theorem 9. For $n \geqslant 2$, we have $P_{n}(x), P_{n}^{+}(x), P_{n}^{-}(x) \in \mathrm{RZ}(-\infty, 0)$ and

$$
P_{n+1}(x) \ll S_{n}(x), P_{n+1}^{+}(x) \prec S_{n}(x), S_{n}(x) \ll P_{n+1}^{-}(x) .
$$

Proof. For $n \geqslant 2$, let $r_{\lfloor n / 2\rfloor}<r_{\lfloor n / 2\rfloor-1}<\cdots<r_{2}<r_{1}$ be the distinct zeros of $S_{n}(x)$. Then by (14), we get $\operatorname{sgn} P_{n+1}\left(r_{i}\right)=(-1)^{i-1}$ for $i=1,2, \ldots,\lfloor n / 2\rfloor$. Hence $P_{n+1}(x)$ has precisely one zero in each of $\lfloor n / 2\rfloor-1$ intervals $\left(r_{\lfloor n / 2\rfloor}, r_{\lfloor n / 2\rfloor-1}\right), \ldots,\left(r_{2}, r_{1}\right)$. Recall that $\operatorname{deg} P_{n+1}(x)=\lfloor n / 2\rfloor$. If $n=2 k+1$ is odd, then $\operatorname{sgn} P_{n+1}\left(r_{k}\right)=(-1)^{k-1}$ and $\operatorname{sgn} P_{n+1}(-\infty)=(-1)^{k}$. If $n=2 k+2$ is even, then $\operatorname{sgn} P_{n+1}\left(r_{k+1}\right)=(-1)^{k}$ and $\operatorname{sgn} P_{n+1}(-\infty)=(-1)^{k+1}$. Thus $P_{n+1}(x)$ has an additional zero in the interval $\left(-\infty, r_{\lfloor n / 2\rfloor}\right)$. Therefore, we have $P_{n+1}(x) \ll S_{n}(x)$. Similarly, by using (12), one can derive $P_{n+1}^{+}(x) \prec S_{n}(x)$ and $S_{n}(x) \ll P_{n+1}^{-}(x)$.

\section{Up-down runs of simsun permutations}

An alternating subsequence of $\pi \in \mathfrak{S}_{n}$ is a subsequence $\pi\left(i_{1}\right), \pi\left(i_{2}\right), \ldots, \pi\left(i_{k}\right)$ satisfying

$$
\pi\left(i_{1}\right)>\pi\left(i_{2}\right)<\pi\left(i_{3}\right)>\cdots \pi\left(i_{k}\right),
$$

where $i_{1}<i_{2}<\cdots<i_{k}$. Motivated by the study of the longest increasing subsequences, Stanley [23] initiated a study of the longest alternating subsequences. Let $\ell_{n}(\pi)$ be the length (number of terms) of the longest alternating subsequence of a permutation $\pi \in \mathfrak{S}_{n}$. The up-down runs of a permutation $\pi$ are the alternating runs of $\pi$ endowed with a 0 in 
the front (see [21, A186370]). For example, the permutation $\pi=514623$ has 4 alternating runs and 5 up-down runs. Let uprun $(\pi)$ be the number of up-down runs of $\pi$. It is clear that uprun $(\pi)=\ell_{n}(\pi)$ for $\pi \in \mathfrak{S}_{n}$. We define

$$
T_{n}(x)=\sum_{\pi \in \mathcal{R} \mathcal{S}_{n}} x^{\mathrm{uprun}(\pi)}=\sum_{k=1}^{n} T(n, k) x^{k} .
$$

The first few terms of $T_{n}(x)$ are

$$
T_{1}(x)=x, T_{2}(x)=x+x^{2}, T_{3}(x)=x+2 x^{2}+2 x^{3}, T_{4}(x)=x+3 x^{2}+8 x^{3}+4 x^{4} .
$$

Theorem 10. For $n \geqslant 1$, the numbers $T(n, k)$ satisfy the recurrence relation

$$
T(n, k)=\lceil k / 2\rceil T(n-1, k)+T(n-1, k-1)+(n-k+1) T(n-1, k-2),
$$

with initial conditions $T(0,0)=1$ and $T(0, k)=0$ for $k>0$ or $k<0$.

Proof. There are three ways in which a permutation $\pi^{\prime} \in \mathcal{R} \mathcal{S}_{n}$ with uprun $\left(\pi^{\prime}\right)=k$ can be obtained from a permutation $\pi \in \mathcal{R} \mathcal{S}_{n-1}$ by inserting the entry $n$ into $\pi$.

(a) If uprun $(\pi)=k$, then we can insert the entry $n$ right after the end of each ascending run. This accounts for $\lceil k / 2\rceil T(n-1, k)$ possibilities.

(b) If uprun $(\pi)=k-1$, then we distinguish two cases: when $\pi$ ends with an ascending run, we insert the entry $n$ to the front of the last entry of $\pi$; when $\pi$ ends with descending run, we insert the entry $n$ at the end of $\pi$. This gives $T(n-1, k-1)$ possibilities.

(c) If uprun $(\pi)=k-2$, then we can insert the entry $n$ into the remaining $n-k+1$ positions. This gives $(n-k+1) T(n-1, k-2)$ possibilities.

This completes the proof of (16).

Note that $S(n, 0)=T(n, 1)=1$, corresponding to the permutation $12 \cdots n$. Recall that an element $\pi$ of $\mathfrak{S}_{n}$ is alternating if $\pi(1)>\pi(2)<\pi(3)>\cdots \pi(n)$. In other words, $\pi(i)<\pi(i+1)$ if $i$ is even and $\pi(i)>\pi(i+1)$ if $i$ is odd. If $n=2 m$ is even, then $S(2 m, m)=T(2 m, 2 m)$, corresponding to the number of alternating permutations in $\mathcal{R S}_{2 m}$. If $n=2 m+1$ is odd, applying the complement operation $\phi$ to $\pi \in \mathcal{R} \mathcal{S}_{n}$, i.e., $\phi(\pi(i))=\pi(n+1-i)$, it is clear that $P(2 m+1, m)=T(2 m+1,2 m+1)$ counts the number of alternating permutations in $\mathcal{R S}_{2 m+1}$. In general, by analyzing permutations in $\mathcal{R S}_{n}^{+}$and $\mathcal{R} \mathcal{S}_{n}^{-}$, it is easy to verify that

$$
S(n, k)=T(n, 2 k)+T(n, 2 k+1), P(n, k)=T(n, 2 k+1)+T(n, 2 k+2) .
$$

Equivalently, we have

$$
(1+x) T_{n}(x)=x S_{n}\left(x^{2}\right)+x^{2} P_{n}\left(x^{2}\right) \text { for } n \geqslant 1 .
$$


Theorem 11. For $n \geqslant 0$, we have

$$
T_{n+1}(x)=x(1+n x) S_{n}\left(x^{2}\right)+\frac{1}{2} x^{2}(1-2 x) S_{n}^{\prime}\left(x^{2}\right) .
$$

Proof. It follows from (3), (14) and (17) that

$$
\begin{aligned}
(1+x) T_{n+1}(x) & =x S_{n+1}\left(x^{2}\right)+x^{2} P_{n+1}\left(x^{2}\right) \\
& =x\left(\left(1+n x^{2}\right) S_{n}\left(x^{2}\right)+\frac{1}{2} x\left(1-2 x^{2}\right) S_{n}^{\prime}\left(x^{2}\right)\right)+x^{2}\left((n+1) S_{n}\left(x^{2}\right)-\frac{x}{2} S_{n}^{\prime}\left(x^{2}\right)\right) \\
& =x\left(1+(n+1) x+n x^{2}\right) S_{n}\left(x^{2}\right)+\frac{1}{2} x^{2}\left(1-x-2 x^{2}\right) S_{n}^{\prime}\left(x^{2}\right) \\
& =x(1+x)(1+n x) S_{n}\left(x^{2}\right)+\frac{1}{2} x^{2}(1+x)(1-2 x) S_{n}^{\prime}\left(x^{2}\right) .
\end{aligned}
$$

The statement immediately follows.

We call the simsun permutations discussed above to be the simsun permutations of the first kind. In the next section, we shall introduce the simsun permutations of the second kind.

\section{Simsun permutations of the second kind}

In this section, we always write $\pi \in \mathfrak{S}_{n}$ in standard cycle decomposition, where each cycle is written with its smallest entry first and the cycles are written in increasing order of their smallest entry. For each $\pi \in \mathfrak{S}_{n}$, we say that $\pi$ has an excedance at $i$ if $\pi(i)>i$. The excedance number of $\pi$ is defined by $\operatorname{exc}(\pi)=\#\{i \in[n-1]: \pi(i)>i\}$. Following [20], for $\pi \in \mathfrak{S}_{n}$, a value $x=\pi(i)$ is called a double excedance if $i=\pi^{-1}(x)<x<\pi(x)$, and we say that $x=\pi(i)$ is a cyclic peak if $i=\pi^{-1}(x)<x>\pi(x)$. Let cpk $(\pi)$ denote the number of cyclic peaks of $\pi$.

Definition 12. We say that $\pi \in \mathfrak{S}_{n}$ is a simsun permutation of the second kind if for all $k \in[n]$, after removing the $k$ largest letters of $\pi$, the resulting permutation has no double excedances.

For example, $(1,5,3,4)(2)$ is not a simsun permutation of the second kind since when we remove the letter 5 , the resulting permutation $(1,3,4)(2)$ contains a double excedance. Let $\mathcal{S S}_{n}$ be the set of the simsun permutations of the second kind of length $n$. It is clear that $\operatorname{exc}(\pi)=\operatorname{cpk}(\pi)$ for $\pi \in \mathcal{S S}_{n}$. We say that $\pi(i)$ is a right-to-left minimum of $\pi$ if $\pi(i)<\pi(j)$ for $j>i$. Let rmin denote the number of right-to-left minima of $\pi$. Let cyc $(\pi)$ be the number of cycles of $\pi$.

In the following, we shall present a constructive proof of the following identity:

$$
\left.\left.\mid\left\{\pi \in \mathcal{R S}_{n}: \operatorname{des}(\pi)=k, \operatorname{rmin}(\pi)=\ell\right)\right\}|=|\left\{\pi \in \mathcal{S S}_{n}: \operatorname{exc}(\pi)=k, \operatorname{cyc}(\pi)=\ell\right)\right\} \mid \text {. }
$$

Let $\mathcal{S S}_{n, k}=\left\{\pi \in \mathcal{S S}_{n} \mid \operatorname{exc}(\pi)=k\right\}$. As a variant of Definition 2, we introduce a definition of the labeled simsun permutations of the second kind. 
Definition 13. Let $\sigma \in \mathcal{S S}_{n, k}$. Suppose $i_{1}<i_{2}<\cdots<i_{k}$ are the excedances of $\sigma$. Then we put the superscript labels $u_{r}$ right after $i_{r}$, where $1 \leqslant r \leqslant k$. In the remaining positions except the first position of each cycle and the positions right after $\sigma\left(i_{r}\right)$, we put the superscript labels $v_{1}, v_{2}, \ldots, v_{n-2 k}$ from left to right.

As an example, for $\sigma=(1,3)(2,4)(5) \in \mathcal{S S}_{5,2}$, the labeling of $\sigma$ is $\left(1^{u_{1}} 3\right)\left(2^{u_{2}} 4\right)\left(5^{v_{1}}\right)$.

Now we start to construct a bijection, denoted by $\Psi$, between $\mathcal{R} \mathcal{S}_{n, k}$ and $\mathcal{S S}_{n, k}$. When $n=1$, we have $\mathcal{R} \mathcal{S}_{1,0}=\left\{{ }^{y_{1}} 1\right\}$. Set $\Psi\left({ }^{y_{1}} 1\right)=\left(1^{v_{1}}\right)$. This gives a bijection between $\mathcal{R} \mathcal{S}_{1,0}$ and $\mathcal{S S}_{1,0}$. Let $n=m$. Suppose $\Psi$ is a bijection between $\mathcal{R S}_{m, k}$ and $\mathcal{S S}_{m, k}$ for all $k$. Given $\pi \in \mathcal{R S}_{m, k}$. Suppose $\Psi(\pi)=\sigma$. Consider the following three cases:

(i) If $\widehat{\pi}$ is obtained from $\pi$ by inserting the entry $m+1$ to the position of $\pi$ with label $x_{r}$, then we insert the entry $m+1$ to $\sigma$ with label $u_{r}$. In this case, $\operatorname{des}(\widehat{\pi})=$ $\operatorname{exc}(\Psi(\widehat{\pi}))=k$. Hence $\widehat{\pi} \in \mathcal{R S}_{m+1, k}$ and $\Psi(\widehat{\pi}) \in \mathcal{S S}_{m+1, k}$.

(ii) If $\widehat{\pi}$ is obtained from $\pi$ by inserting the entry $m+1$ to the position of $\pi$ with label $y_{r}$, then we insert $m+1$ to $\sigma$ with label $v_{r}$. In this case, $\operatorname{des}(\widehat{\pi})=\operatorname{exc}(\Psi(\widehat{\pi}))=k+1$. Hence $\widehat{\pi} \in \mathcal{R S}_{m+1, k+1}$ and $\Psi(\widehat{\pi}) \in \mathcal{S S}_{m+1, k+1}$.

(iii) If $\widehat{\pi}$ is obtained from $\pi$ by inserting the entry $m+1$ at the end of $\pi$, then we append $(m+1)$ to $\sigma$ as a new cycle. Hence $\widehat{\pi} \in \mathcal{R S}_{m+1, k}$ and $\Psi(\widehat{\pi}) \in \mathcal{S S}_{m+1, k}$. From this case, we see that $\operatorname{rmin}(\widehat{\pi})=\operatorname{rmin}(\pi)+1$ if and only if cyc $(\Psi(\widehat{\pi}))=\operatorname{cyc}(\sigma)+1$.

By induction, we see that $\Psi$ is the desired bijection between $\mathcal{R} \mathcal{S}_{m, k}$ and $\mathcal{S S}_{m, k}$ for all $k$, which also gives a constructive proof of (18).

Example 14. Given $\pi=3412 \in \mathcal{R S}_{4,1}$. The correspondence between $\pi$ and $\Psi(\pi)$ is built up as follows:

$$
\begin{aligned}
y_{1} & \Leftrightarrow\left(1^{v_{1}}\right) ; \\
{ }^{y_{1}} 1^{y_{2}} 2 & \Leftrightarrow\left(1^{v_{1}}\right)\left(2^{v_{2}}\right) ; \\
3^{x_{1}} 1^{y_{1}} 2 & \Leftrightarrow\left(1^{u_{1}} 3\right)\left(2^{v_{1}}\right) ; \\
y_{1} 34^{x_{1}} 1^{y_{2}} 2 & \Leftrightarrow\left(1^{u_{1}} 43^{v_{1}}\right)\left(2^{v_{2}}\right) .
\end{aligned}
$$

Consider the following enumerative polynomials

$$
S_{n}(x, q)=\sum_{\pi \in \mathcal{S} \mathcal{S}_{n}} x^{\operatorname{exc}(\pi)} q^{\mathrm{cyc}(\pi)} .
$$

Let $S=S(x, q ; z)=\sum_{n \geqslant 0} S_{n}(x, q) \frac{z^{n}}{n !}$. We now present the main result of this section.

Theorem 15. The polynomials $S_{n}(x, q)$ satisfy the recurrence relation

$$
S_{n+1}(x, q)=(q+n x) S_{n}(x, q)+x(1-2 x) \frac{\partial}{\partial x}\left(S_{n}(x, q)\right)
$$

with the initial condition $S_{0}(x, q)=1$. Furthermore,

$$
S(x, q ; z)=R S^{q}(x, z) .
$$


Proof. Let $n$ be a fixed positive integer and given $\sigma \in \mathcal{S S}_{n}$. Let $\sigma_{i}$ be an element of $\mathcal{S S}_{n+1}$ obtained from $\sigma$ by inserting the entry $n+1$, in the standard cycle decomposition of $\sigma$, right after $i$ if $i$ is not a cyclic peak of $\sigma$ and $i \in[n]$ or as a new cycle $(n+1)$ if $i=n+1$. It is clear that

$$
\operatorname{cyc}\left(\sigma_{i}\right)= \begin{cases}\operatorname{cyc}(\sigma), & \text { if } i \in[n] \\ \operatorname{cyc}(\sigma)+1, & \text { if } i=n+1\end{cases}
$$

Therefore, we have

$$
\begin{aligned}
S_{n+1}(x, q) & =\Sigma_{\pi \in \mathcal{S} \mathcal{S}_{n+1}} x^{\operatorname{exc}(\pi)} q^{\operatorname{cyc}(\pi)} \\
& =\Sigma_{i=1}^{n+1} \Sigma_{\sigma \in \mathcal{S} \mathcal{S}_{n}} x^{\operatorname{exc}\left(\sigma_{i}\right)} q^{\operatorname{cyc}\left(\sigma_{i}\right)} \\
& =\Sigma_{\sigma \in \mathcal{S} \mathcal{S}_{n}} x^{\operatorname{exc}(\sigma)} q^{\operatorname{cyc}(\sigma)+1}+\Sigma_{i=1}^{n} \Sigma_{\sigma \in \mathcal{S S}} x^{\operatorname{exc}\left(\sigma_{i}\right)} q^{\operatorname{cyc}(\sigma)} \\
& =q S_{n}(x, q)+\Sigma_{\sigma \in \mathcal{S} \mathcal{S}_{n}}\left(\operatorname{exc}(\sigma) x^{\operatorname{exc}(\sigma)}+(n-2 \operatorname{exc}(\sigma)) x^{\operatorname{exc}(\sigma)+1}\right) q^{\operatorname{cyc}(\sigma)} \\
& =q S_{n}(x, q)+n x S_{n}(x, q)+\Sigma_{\pi \in \mathcal{S S}_{n}}(1-2 x) \operatorname{exc}(\sigma) x^{\operatorname{exc}(\sigma)} q^{\operatorname{cyc}(\sigma)},
\end{aligned}
$$

and (19) follows. By rewriting (19) in terms of the generating function $S$, we have

$$
(1-x z) S_{z}=q S+x(1-2 x) S_{x} .
$$

It is routine to check that the generating function $\widetilde{S}=\widetilde{S}(x, q ; z)=R S^{q}(x, z)$ satisfies $(21)$. Also, this generating function gives $\widetilde{S}(x, q ; 0)=1, \widetilde{S}(x, 0 ; z)=1$ and $\widetilde{S}(0, q ; z)=e^{q z}$. Hence $S=\widetilde{S}$.

Combining (19) and [19, Theorem 2], we get the following corollary.

Corollary 16. If $q>0$, then $S_{n}(x, q)$ has nonpositive and simple zeros for $n \geqslant 2$.

Using (20), it is easy to verify that

$$
S(1, q ; z)=\frac{1}{(1-\sin z)^{q}} .
$$

and for $n \geqslant 1$, we have

$$
S_{n}(x,-1)= \begin{cases}(1-x)(1-2 x)^{m-1}, & \text { if } n=2 m \\ -(1-2 x)^{m}, & \text { if } n=2 m+1 .\end{cases}
$$

A cycle $(b(1), b(2), \ldots)$ is said to be up-down if it satisfies $b(1)<b(2)>b(3)<\cdots$, where $b_{1}$ is the smallest element of this cycle. We say that a permutation $\pi$ is cycle-updown if it is a product of up-down cycles. Let $\triangle_{n}$ be the set of cycle-up-down permutations in $\mathfrak{S}_{n}$. Deutsch and Elizalde [7, p. 193] discovered that

$$
\sum_{n \geqslant 0} \sum_{\pi \in \triangle_{n}} q^{\operatorname{cyc}(\pi)} \frac{z^{n}}{n !}=S(1, q ; z) .
$$


Therefore, we have

$$
\sum_{\pi \in \mathcal{S S}_{n}} q^{\mathrm{cyc}(\pi)}=\sum_{\pi \in \triangle_{n}} q^{\mathrm{cyc}(\pi)}
$$

Let fix $(\pi)$ be the number of fixed points of $\pi$, i.e., fix $(\pi)=|\{i \in[n]: \pi(i)=i\}|$. Define

$$
F_{n}(x, y, q)=\sum_{\pi \in \mathcal{S} \mathcal{S}_{n}} x^{\operatorname{exc}(\pi)} y^{\text {fix }(\pi)} q^{\mathrm{cyc}(\pi)} .
$$

Note that

$$
\begin{aligned}
F_{n}(x, y, q) & =\sum_{i=0}^{n}\left(\begin{array}{c}
n \\
i
\end{array}\right)(y q-q)^{i} \sum_{\pi \in \mathcal{S S}_{n-i}} x^{\operatorname{exc}(\pi)} q^{\mathrm{cyc}(\pi)} \\
& =\sum_{i=0}^{n}\left(\begin{array}{c}
n \\
i
\end{array}\right)(y q-q)^{i} S_{n-i}(x, q) .
\end{aligned}
$$

Using (20), we obtain

$$
\sum_{n \geqslant 0} F_{n}(x, y, q) \frac{z^{n}}{n !}=e^{q z(y-1)} R S^{q}(x, z) .
$$

\section{Concluding remarks}

In this paper we study the peak statistics on simsum permutations. It is well known that the descent statistic is equidistributed over $n$-simsun permutations and $n$-André permutations (see [5]), and there are bijections between simsun permutations and increasing 1-2 trees (see [6] for instance). Therefore, one can find corresponding results on André permutations and increasing 1-2 trees. For example, $S(1, q ; z)$ also is the (shifted) exponential generating function that counts André permutations with respect to the size and the number of right-to-left minima (see [9, Proposition 1]). Furthermore, it would be interesting to derive similar results on signed simsum permutations introduced by Ehrenborg and Readdy [10].

\section{Acknowledgements}

The authors thank the referees for their valuable suggestions which lead to a substantial improvement of the paper.

\section{References}

[1] D. André. Étude sur les maxima, minima et séquences des permutations. Ann. Sci. École Norm. Sup., 3(1):121-135, 1884.

[2] S. Billey, K. Burdzy, B.E. Sagan. Permutations with given peak set. J. Integer Seq., 16: Article 13.6.1, 2013. 
[3] P. Brändén and A. Claesson. Mesh patterns and the expansion of permutation statistics as sums of permutation patterns. Electron. J. Combin., 18(2): \#P5, 2011.

[4] W.Y.C. Chen, N.J.Y. Fan, J.Y.T. Jia. Labeled ballot paths and the Springer numbers. SIAM J. Discrete Math., 25:1530-1546, 2011.

[5] C-O. Chow, W. C. Shiu. Counting simsun permutations by descents. Ann. Comb., 15:625-635, 2011.

[6] W.-C. Chuang, S.-P. Eu, T.-S. Fu, Y.-J. Pan. On simsun and double simsun permutations avoiding a pattern of length three. Fund. Inform., 117:155-177, 2012.

[7] E. Deutsch and S. Elizalde. Cycle-up-down permutations. Australas. J. Combin., 50:187-199, 2011.

[8] E. Deutsch, S. Elizalde. Restricted simsun permutations. Ann. Combin., 16(2):253269, 2012.

[9] F. Disanto. André permutations, right-to-left and left-to-right minima. Séminaire Lothar. Combin., 70:Article B70f, 2014.

[10] R. Ehrenborg, M. Readdy. Coproducts and the cd-index. J. Algebraic Combin., 8:273-299, 1998.

[11] S.-P. Eu, T.-S. Fu, Y.-J. Pan. A refined sign-balance of simsun permutations. European J. Combin., 36:97-109, 2014.

[12] D. Foata and M.P. Schützenberger. Nombres d'Euler et permutations alternantes, in A Survey of Combinatorial Theory, J.N. Srivastava et al. (Eds.), Amsterdam, North-Holland, pages 173-187, 1973.

[13] D. Foata, G.-N. Han. Arbres minimax et polynômes d'André. Adv. in Appl. Math., $27: 367-389,2001$.

[14] J. Garloff, D.G. Wagner. Hadamard products of stable polynomials are stable. J. Math. Anal. Appl., 202:797-809, 1996.

[15] G. Hetyei, E. Reiner. Permutation trees and variation statistics. European J. Combin., 19:847-866, 1998.

[16] S.-M. Ma. Derivative polynomials and enumeration of permutations by number of interior and left peaks. Discrete Math., 312:405-412, 2012.

[17] S.-M. Ma. An explicit formula for the number of permutations with a given number of alternating runs. J. Combin. Theory Ser. A, 119:1660-1664, 2012.

[18] S.-M. Ma. Enumeration of permutations by number of alternating runs. Discrete Math., 313:1816-1822, 2013.

[19] S.-M. Ma, Y. Wang. $q$-Eulerian polynomials and polynomials with only real zeros. Electron. J. Combin., 15:R17, 2008.

[20] H. Shin, J. Zeng. The symmetric and unimodal expansion of Eulerian polynomials via continued fractions. European J. Combin. 33:111-127, 2012.

[21] N.J.A. Sloane. The On-Line Encyclopedia of Integer Sequences, published electronically at http://oeis.org, 2010. 
[22] T.A. Springer. Remarks on a combinatorial problem. Nieuw Arch. Wisk., 19:30-36, 1971.

[23] R.P. Stanley. Longest alternating subsequences of permutations. Michigan Math. J., 57:675-687, 2008.

[24] S. Sundaram. The homology representations of the symmetric group on CohenMacaulay subposets of the partition lattice. Adv. Math., 104(2):225-296, 1994.

[25] S. Sundaram. The homology of partitions with an even number of blocks. J. Algebraic Combin., 4:69-92, 1995.

[26] S. Sundaram. Plethysm, partitions with an even number of blocks and Euler numbers. DIMACS Series in Discrete Mathematics and Theoretical Computer Science 24, (Billera, Greene, Simion, Stanley, eds.), Amer. Math. Soc., 171-198, 1996. 